Research Article

\title{
Electronic, Structural, and Optical Properties of Zinc Blende and Wurtzite Cadmium Sulfide (CdS) Using Density Functional Theory
}

\author{
Teshome Gerbaba Edossa ${ }^{1}{ }^{1}$ and Menberu Mengasha Woldemariam (iD ${ }^{2}$ \\ ${ }^{1}$ Department of Physics, Wollega University, P. O. Box 395, Nekemte, Ethiopia \\ ${ }^{2}$ Department of Physics, Jimma University, P. O. Box 378, Jimma, Ethiopia \\ Correspondence should be addressed to Teshome Gerbaba Edossa; teshgerb19@gmail.com and Menberu \\ Mengasha Woldemariam; menberu.mengesha@ju.edu.et
}

Received 28 May 2020; Revised 26 June 2020; Accepted 29 June 2020; Published 20 August 2020

Academic Editor: Charles Rosenblatt

Copyright (c) 2020 Teshome Gerbaba Edossa and Menberu Mengasha Woldemariam. This is an open access article distributed under the Creative Commons Attribution License, which permits unrestricted use, distribution, and reproduction in any medium, provided the original work is properly cited.

\begin{abstract}
Zinc blende $(\mathrm{zb})$ and wurtzite $(\mathrm{wz})$ structure of cadmium sulfide (CdS) are analyzed using density functional theory within local density approximation (LDA), generalized gradient approximation (GGA), Hubbard correction (GGA + U), and hybrid functional approximation (PBE0 or HSE06). To assure the accuracy of calculation, the convergence test of total energy with respect to energy cutoff and $k$-point sampling is performed. The relaxed atomic position for the $\mathrm{CdS}$ in $\mathrm{zb}$ and wz structure is obtained by using total energy and force minimization method following the Hellmann-Feynman approach. The structural optimization and electronic band structure properties of CdS are investigated. Analysis of the results shows that LDA and GGA underestimate the bandgap due to their poor approximation of exchange-correlation functional. However, the Hubbard correction to GGA and the hybrid functional approximation give a good bandgap value which is comparable to the experimental result. Moreover, the optical properties such as real and imaginary parts of the dielectric function, the absorption coefficient, and the energy loss function of CdS are determined.
\end{abstract}

\section{Introduction}

Chalcogenide is a chemical compound consisting of at least one chalcogen anion and one more electropositive element. Hence, the term chalcogenide is more commonly reserved for sulfides, selenides, and tellurides rather than oxides [1]. Cadmium chalcogenides are monochalcogenides having the formula $\mathrm{CdX}(\mathrm{X}=\mathrm{S}, \mathrm{Se}$, and $\mathrm{Te})$. They typically crystallize in one of the three motifs, zinc blende (zb) (face-centered cubic), wurtzite (wz) (hexagonal), and rock salt (cubic) structures [2]. The experimentally stable crystal phase of CdS is a wurtzite structure [3]. Furthermore, the CdS can crystallize in either zinc blende (zb) crystal structure with space group F-43m or wurtzite (wz) crystal structure with $\mathrm{P}_{3}$ mc space group under ambient conditions [4]. Moreover, in a recent experimental measurement report, CdS phase transition occurs from $\mathrm{zb}$ to $\mathrm{wz}$ phase between pressure values of $3.0 \mathrm{GPa}$ and $4.3 \mathrm{GPa}$ [4-6]. The rock salt structure is only observed under high pressure $[6,7]$.

Cadmium chalcogenides $\mathrm{CdX}(\mathrm{X}=\mathrm{S}$, Se, and $\mathrm{Te})$ and their combinations are widely studied members of group II-VI semiconductor family [8, 9]. Thin films of cadmium chalcogenides have received intensive attention due to their application in solar cells, photoelectrochemical cells, IR detectors, lux meters, switching devices, and Schottky barriers $[10,11]$. The experimental bandgap of wz-CdS thin film is $2.42 \mathrm{eV}$ using a chemical bath technique [12]. Photoluminescence measurements of $\mathrm{zb}$-CdS lead to a bandgap of $2.4 \mathrm{eV}$ [13]. The spectroscopic ellipsometry study of $\mathrm{zb}$ $\mathrm{CdS}$ gives a bandgap of $2.4 \mathrm{eV}$ [14]. However, the bandgap calculated using DFT is $1.05 \mathrm{eV}$ [15], $1.2 \mathrm{eV}$ [16], and $1.22 \mathrm{eV}$ [17] which is underestimated compared with the experimental value. The challenging issue with semilocal functional such as LDA and GGA is that they consist of spurious 
electron self-interaction energy [18]. This interaction results with considerable errors in reaction energies for which electrons are transferred between significantly different environments such as a metal and a transition metal oxide. One popular approach that used to overcome this problem is to use a so-called Hubbard U parameter that will cancel part of the self-interaction energy orbitals on the most problematic orbitals such as localized $-\mathrm{d}$ or $-\mathrm{f}$ orbitals $[10,19,20]$. Moreover, hybrid density functional which incorporates a certain amount of Hartree-Fock (HF) exchange has further improved upon PBE results [21]. This improvement apparently comes in the inclusion of nondynamical correlation which effectively delocalizes the PBE exchange hole. Successful hybrid calculations of solids are possible using Gaussian-type orbitals and periodic boundary conditions. To obtain an appropriate bandgap of CdS, $\mathrm{DFT}+\mathrm{U}$ approach and the exchange-correlation functional with hybrid functions are natural choices for open shell d-block metals. The optical bandgaps of these materials lie close to the range of optimum theoretically achievable energy conversion efficiency [22]. In this study, we carefully examined the structural, electronic, and optical properties of $\mathrm{CdS}$ in zinc blende and wurtzite structures using density functional theory.

\section{Computational Method}

The computations are carried out using Quantum ESPRESSO (QE) package that is based on DFT and planewave pseudopotential method [23, 24]. In Kohn-Sham equations, the exchange-correlation potential is unknown and a challenging term in DFT. To approximate this potential, LDA [25], PBE [26], GGA + U [27], and hybrid (PBE0) [28] functionals are adopted. While approximating this potential, the core electrons which do not participate in the chemical bonding of the system are frozen and only valence electrons are considered. The electron configuration of cadmium is $[\mathrm{Kr}] 4 \mathrm{~d}^{10} 5 \mathrm{~s}^{2}$ and that of sulfur is $[\mathrm{Ne}] 3 \mathrm{~s}^{2} 3 \mathrm{p}^{4}$. In order to treat the strong electron-electron correlation among the d-electrons of cadmium, introducing the Hubbard correction $U$ term improves the approximation. For convergence tests, the electronic wave functions are expanded in a plane-wave basis set with trial cutoff energy. The $k$-point sampling of the Brillouin zone was constructed using Monkhorst and Pack mesh scheme [29]. Convergence test of total energy with respect to energy cutoff and $k$-point sampling is performed to ensure the accuracy of the calculations until the change in energy is equal to $0.01 \mathrm{eV}$. It is observed that $5 \times 5 \times 5$ and $6 \times 6 \times 6 k$-point samplings for Brillouin zone integration yield accurately converged total energy of zb-CdS for DFT $+\mathrm{U}$ and LDA, PBE, and PBE0, respectively. The obtained value of cutoff energy for total energy convergence of zb-CdS is $60 \mathrm{Ry}$ with respect to LDA, $\mathrm{PBE}, \mathrm{PBE} 0$, and $\mathrm{DFT}+\mathrm{U}$ approximations. In case of $\mathrm{wz}-$ $\mathrm{CdS}$, the total energy converged at $6 \times 6 \times 6 k$-point sampling for Brillouin zone integration in relation to LDA and $5 \times 5 \times 5$ in relation to $\mathrm{PBE}, \mathrm{PBE} 0$, and $\mathrm{DFT}+\mathrm{U}$. Moreover, the total energy converged at 55 Ry for LDA and PBE and at 60 Ry for DFT + U and PBE0, respectively.

\section{Result and Discussion}

3.1. Electronic Structure of CdS in $z b$ and $w z$ Phases. Crystal structure is the most important aspect to understand many properties of materials. CdS is group IIB-VI semiconductor which exists in $\mathrm{zb}$ and $\mathrm{wz}$ phases [4]. The wz crystal structure is a member of the hexagonal crystal system, which consists of two interpenetrating hexagonal closepacked (HCP) sublattices, one of atom $A$ and the other of atom $\mathrm{B}$, displaced from each other by $3 / 8 c$ along the $c$-axis. The $\mathrm{zb}$ phase of $\mathrm{CdS}$ consists of two interpenetrating facecentered cubic (FCC) sublattices, one of atom A and the other of atom B, located from each other along the body diagonal by $a / 4$, where $a$ is the lattice constant.

The equilibrium lattice constants of $\mathrm{CdS}$ for both phases were calculated using plane-wave self-consistent field (PWSCF). The calculated values of lattice parameters using LDA and PBE methods are shown in Table 1 and compared with the previous theoretical and experimental results. It shows that our calculations in accordance with LDA and PBE are in good agreement with the previous theoretical and experimental results. LDA underestimates the lattice constant by $1 \%$ whereas PBE overestimates it. Moreover, the graph of lattice constant versus total energy is displayed in Figure 1.

3.2. Band Structure and Total Density of States of CdS. The electronic band structures are calculated along the high symmetry direction of the Brillouin zone using LDA, PBE, $\mathrm{PBE}+\mathrm{U}$, and hybrid functions (PBE0). The band structures for all potential approximations are demonstrated in Figure 2. From the calculations, the obtained bandgap values in $\mathrm{eV}$ with respect to $\mathrm{LDA}, \mathrm{PBE}, \mathrm{PBE}+\mathrm{U}$, and $\mathrm{PBE} 0$ are given in Table 2 and comparision is made with existing theoretical and experimental results.

From Table 2 and Figures 2(a) and 2(b), one can observe that DFT method based on the exchange-correlation functional of LDA and GGA provides underestimated value of the energy bandgap $(\sim 0.741-1.04 \mathrm{eV})$ compared to the experimental energy bandgap. However, the energy bandgap values obtained with respect to $\mathrm{LDA}+\mathrm{U}$ and hybrid functional (PBE0) is in good agreement with the experimental value for $\mathrm{zb}$ and $\mathrm{wz}$ phases.

The density of states for both phases was calculated within LDA, PBE, and DFT $+\mathrm{U}$ as shown in Figures 3(a) and 3(b). Density of states (DOS) helps to understand the behavior of state occupancy over specific energy interval. It provides detail of the states which are unoccupied and the states which are occupied. A high DOS at a specific energy level describes the states available for occupation. However, there is no state occupied at DOS equal to zero. For both phases, the density of states is discontinuous for the width from the top of the valence band to the bottom of the conduction band which is normally the bandgap of the system.

3.3. Optical Properties of CdS. CdS is a semiconductor which has wide application in recent technology due to its exceptional physical properties. It has applications in the field of photonics, energy device, and light sensing. The dielectric 
TABLE 1: Comparison of the calculated and experimental lattice constants.

\begin{tabular}{|c|c|c|c|c|c|}
\hline & & LDA & PBE & $\mathrm{GGA}+\mathrm{U}$ & PBE0 \\
\hline \multicolumn{6}{|c|}{ Phase } \\
\hline \multirow{3}{*}{$\mathrm{zb}$} & Present & $a=5.819$ & $a=5.979$ & $a=5.847$ & $a=5.983$ \\
\hline & Theory & $a=5.81[9], 5.85[30], 5.80[31]$ & & & \\
\hline & Experiment & $a=5.82[32]$ & & & \\
\hline \multirow{4}{*}{ wz } & Present & $a=4.158$ & $a=4.226$ & $a=4.37$ & $a=4.160$ \\
\hline & Present & $c=6.752$ & $c=6.863$ & $c=7.10$ & $c=6.756$ \\
\hline & Theory & $\begin{array}{c}a=4.097[9], a=4.160[30] \\
c=6.752, c=6.70\end{array}$ & & & \\
\hline & Experiment & $\begin{array}{c}a=4.136[32] \\
c=6.756\end{array}$ & & & \\
\hline
\end{tabular}

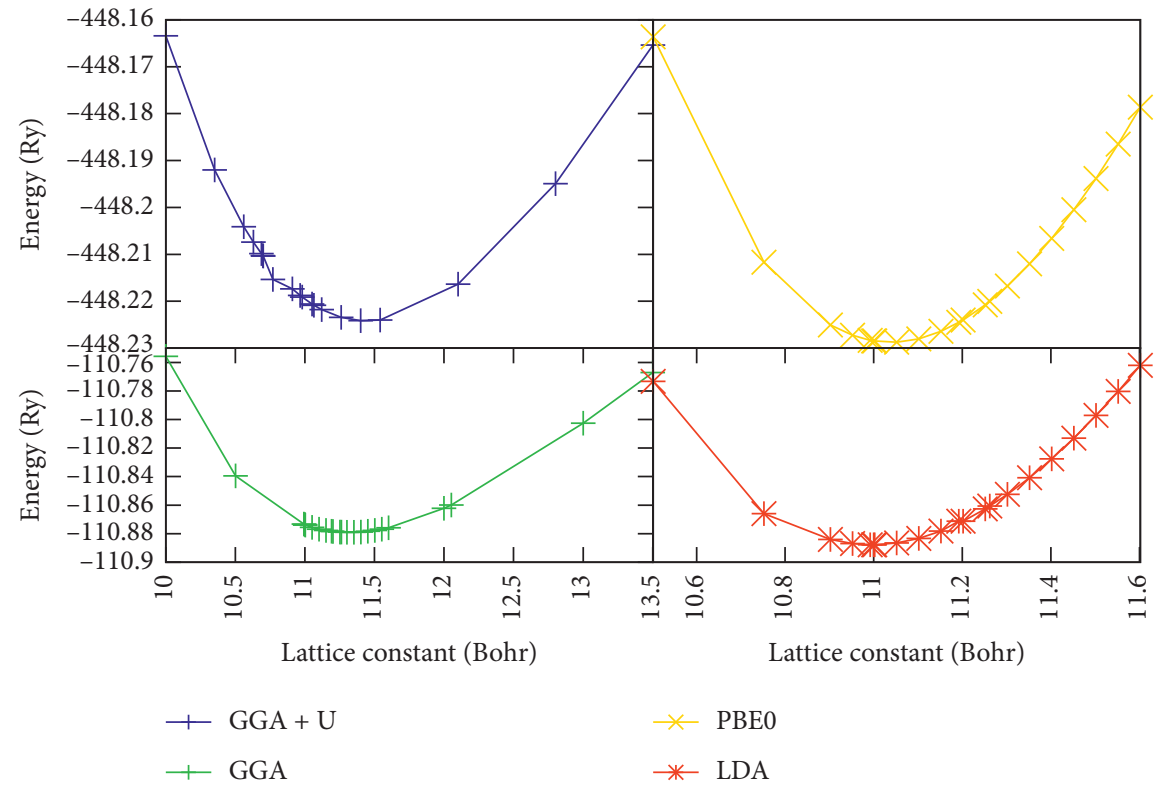

(a)

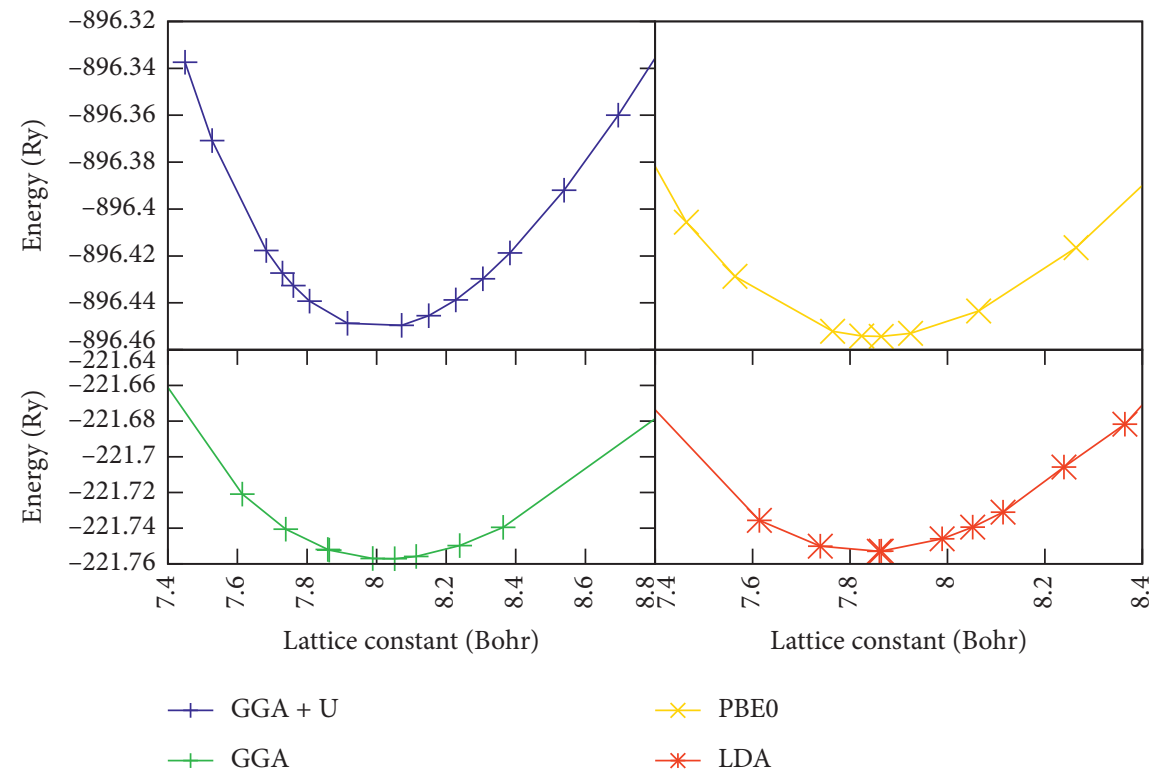

(b)

Figure 1: Lattice constant versus total energy of CdS in zb (a) and wz (b) phases. 

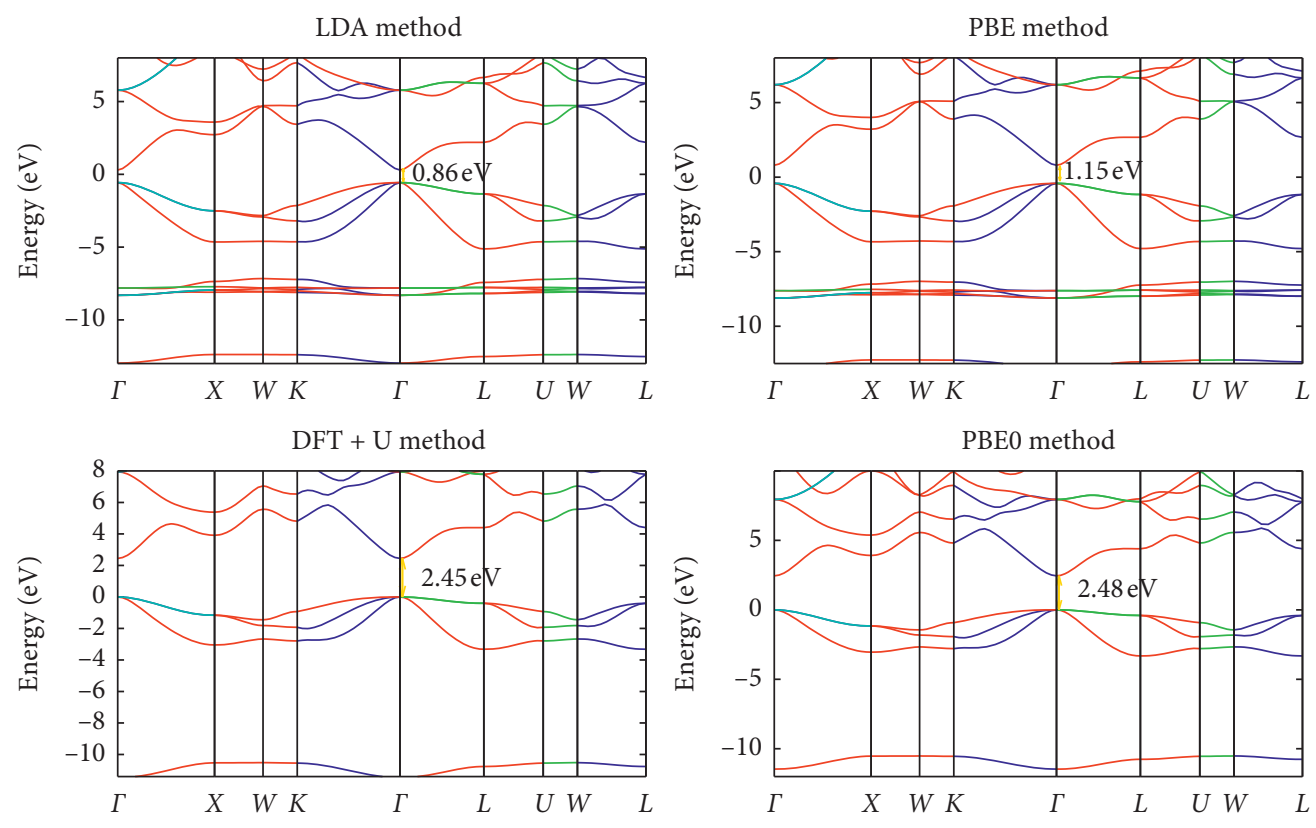

(a)
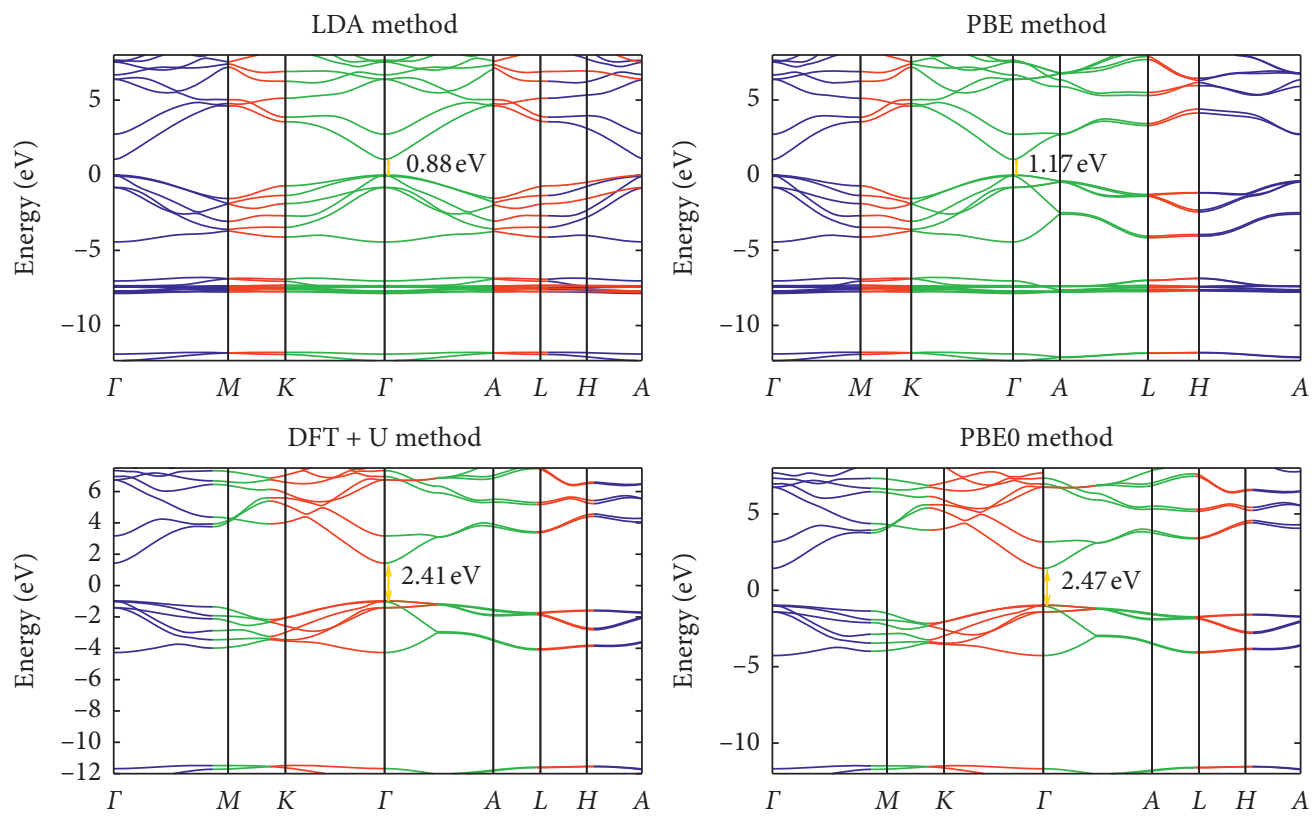

(b)

FIgURE 2: Band structure of (a) zb-CdS structure and (b) wz-CdS structure along high symmetry points with respect to LDA, PBE, PBE + U, and PBE0 approximations.

constant and extinction coefficient are the optical properties of a medium which can be derived from its complex dielectric function, $\varepsilon=\varepsilon_{1}(\omega)+i \varepsilon_{2}(\omega)$. Direct computation of many-body wave function yields the imaginary part of dielectric function [38]:

$$
\varepsilon_{2}(\omega)=\frac{2 e^{2} \pi}{\Omega \varepsilon_{0}} \sum_{k, v, c}\left|\left\langle\varphi_{k}^{c}|r \widehat{\sigma}| \varphi_{k}^{v}\right\rangle\right|^{2} \delta\left(E_{c}^{k}-E_{v}^{k}-\hbar \omega\right)
$$

where $\widehat{\sigma}$ is the vector defining the polarization of incident electric field, $\omega$ is the incident photon frequency, $e$ is the electrostatic charge, and $\Omega$ is the unit cell volume. The superscripts $c$ and $v$ represent the conduction and valence band wave functions. Using the Kramers-Kronig relation [36], the real part of the dielectric function is given by

$$
\varepsilon_{1}(\omega)=1+\frac{4}{\pi} P \int_{0}^{\infty} \frac{\omega \varepsilon_{2}(\omega)}{\omega^{2}-\omega^{2}} d \omega .
$$

The absorption coefficient and the loss function in terms of the real and imaginary parts of dielectric function are given by 


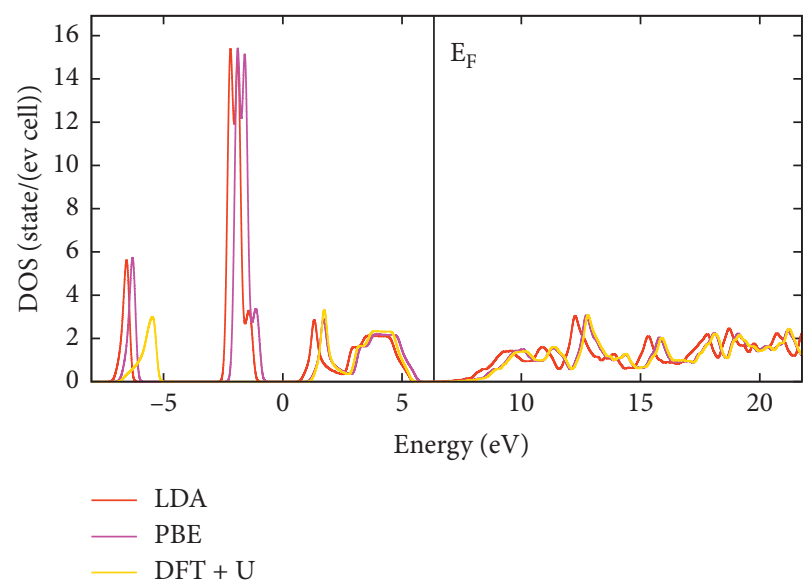

(a)

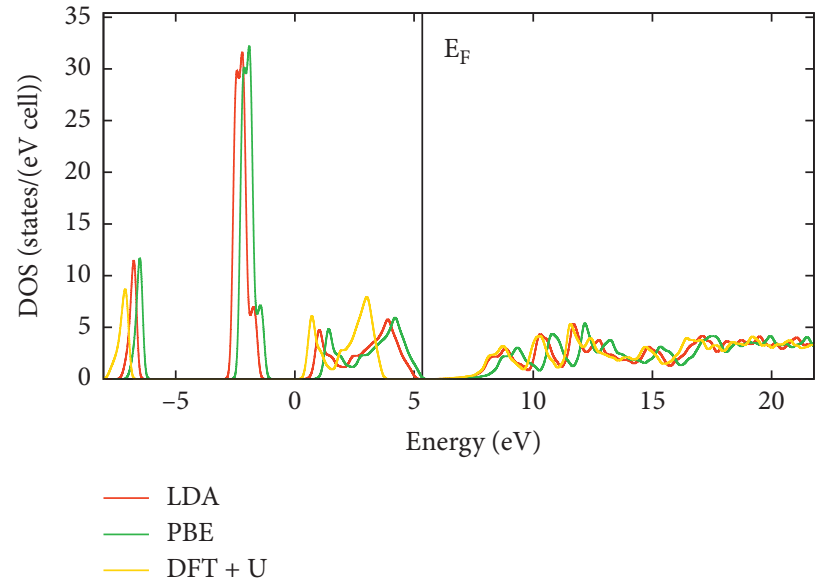

(b)

Figure 3: Total density of states of (a) zb-CdS and (b) wz-CdS.

TABLe 2: Comparison of the calculated and experimental bandgap.

\begin{tabular}{|c|c|c|c|c|c|c|}
\hline Methods & & LDA & PBE & $\mathrm{DFT}+\mathrm{U}$ & PBE0 & \\
\hline \multicolumn{7}{|l|}{ Phase } \\
\hline \multirow{3}{*}{$\mathrm{zb}$} & Present & \multirow{3}{*}{0.86} & 1.15 & \multirow{3}{*}{2.45} & \multirow{3}{*}{2.48} & \multirow{3}{*}{$\begin{array}{c}\text { Exper. } 2.37[34] \\
2.58[36]\end{array}$} \\
\hline & Theory & & $1.37[33]$ & & & \\
\hline & & & 1.24 [35] & & & \\
\hline \multirow{3}{*}{ wz } & Present & \multirow{3}{*}{0.88} & 1.17 & \multirow{3}{*}{2.41} & \multirow{3}{*}{2.47} & \multirow{3}{*}{$\begin{array}{c}\text { Exper. } 2.48[37] \\
2.55[35]\end{array}$} \\
\hline & Theory & & $1.20[16]$ & & & \\
\hline & neory & & 1.15 [35] & & & \\
\hline
\end{tabular}

$$
\begin{aligned}
& \beta(\omega)=\sqrt{2} \omega\left[\sqrt{\varepsilon_{1}(\omega)^{2}+\varepsilon_{2}(\omega)^{2}}-\varepsilon_{1}(\omega)\right]^{\frac{1}{2}}, \\
& L(\omega)=\frac{\varepsilon_{2}(\omega)}{\varepsilon_{1}(\omega)^{2}+\varepsilon_{2}(\omega)^{2}} .
\end{aligned}
$$

The frequency-dependent real $\left(\varepsilon_{1}(\omega)\right)$ and imaginary $\left(\varepsilon_{2}(\omega)\right)$ parts of the dielectric constant of zb- and wz-CdS compounds are studied using TD-DFPT with a help of the Lanczos chain as demonstrated in Figure 4.

Several peaks corresponding to different electronic transitions were observed in the spectra of real and imaginary parts of the dielectric constant. The real part of the dielectric function $\varepsilon_{1}(\omega)$ describes the strength of dynamical screening and the polarization effects. However, the imaginary part of the dielectric function $\varepsilon_{2}(\omega)$ is related to the energy absorption due to charge excitations. The optical properties of the dielectric function of $\mathrm{zb}$ - and wz-CdS are calculated for energy ranging from 0 to $20 \mathrm{eV}$. The peaks seen in the optical spectra are described based on transitions from the occupied to unoccupied bands in the electronic energy band structure, particularly at high symmetry points in the Brillouin zone. The oscillatory trend in this spectrum represents the presence of many-body interactions and it is dependent on the strength of transition. This may be due to the random orientation of transition dipoles with respect to the exciting electromagnetic field direction. For both phases, the real part of the dielectric function has its maxima, when the imaginary part of the dielectric function has its minima.

From Figure 4(a), one can see that the real part of dielectric function $\varepsilon_{1}(\omega)$ of $\mathrm{zb}$-CdS structure has a maximum peak of 14.52 at a photon energy of $0.44 \mathrm{eV}$ using LDA approximation. According to PBE approximation, it has maxima at 10.41 for a photon energy of $0.88 \mathrm{eV}$. Moreover, the static dielectric constant $\varepsilon(0)$ is equal to 12.30 and 7.96 using LDA and PBE approximations, respectively, for $\mathrm{zb}$ $\mathrm{CdS}$ crystal. The peak magnitudes of the imaginary part of the dielectric function for $\mathrm{zb}-\mathrm{CdS}$ is 12.07 at a photon energy of $0.66 \mathrm{eV}$ using the LDA method. However, it has a peak value of 9.76 at a photon energy of $5.94 \mathrm{eV}$ using the PBE method.

From Figure 4(b), it can be demonstrated that the real part of dielectric function $\varepsilon_{1}(\omega)$ of wz-CdS structure has maxima of 7.61 for a photon energy of $0.22 \mathrm{eV}$ based on LDA approximation. However, it has a peak value of 7.64 at a photon energy of $0.22 \mathrm{eV}$ with respect to the PBE method. In addition, our calculation shows that the static dielectric constant $\varepsilon(0)$ of wz-CdS is 7.96 in LDA and 7.3160 in PBE approximation. The maximum value of the imaginary part of dielectric function $\varepsilon_{2}(\omega)$ of wz-CdS is 6.04 at a photon energy of $0.88 \mathrm{eV}$ using the LDA method. However, its value is 6.50 at a photon energy of $1.10 \mathrm{eV}$ in accordance with PBE approximation. The figures clearly confirm that when the real part of the dielectric function has its maxima, the 


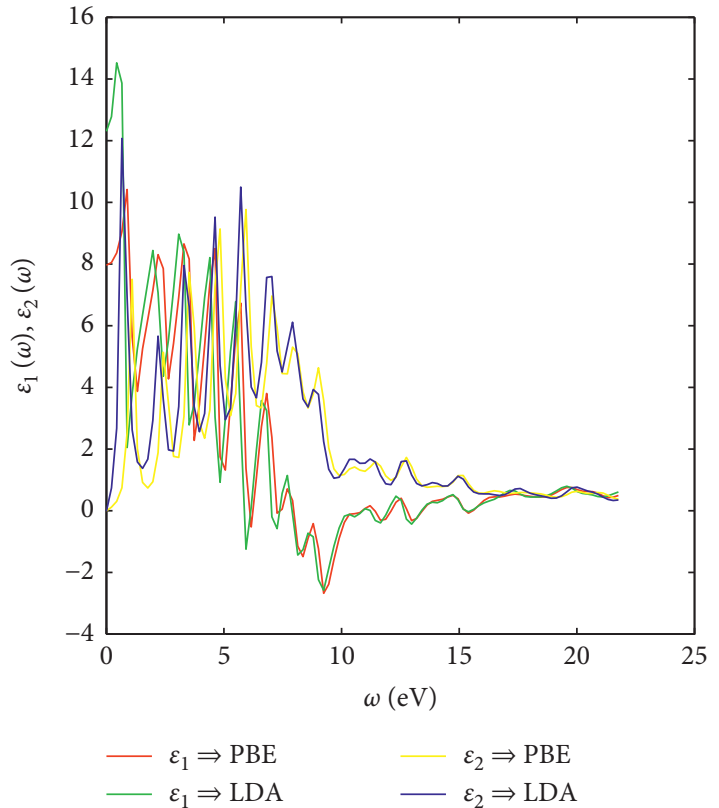

(a)

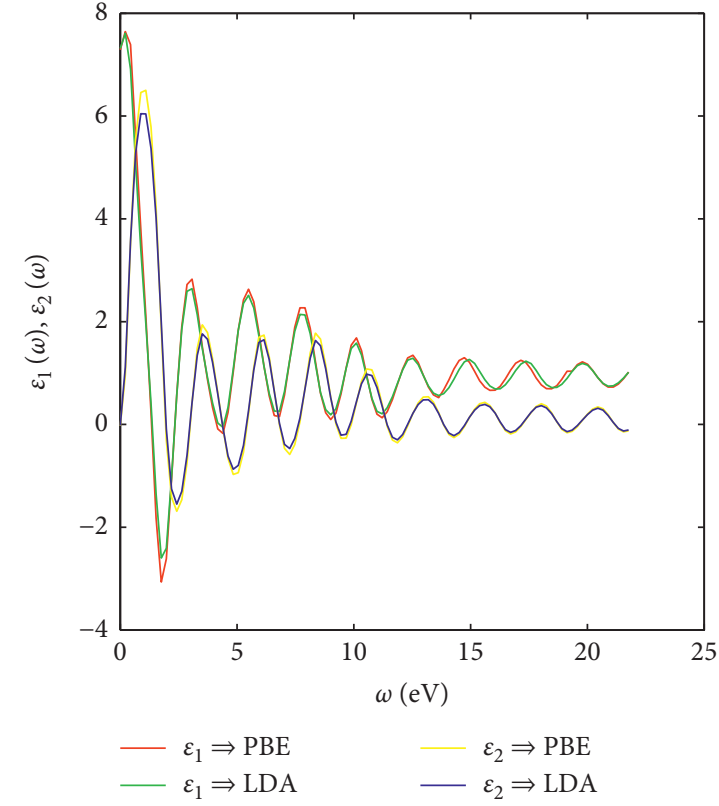

(b)

Figure 4: Real and imaginary parts of dielectric function for (a) zb-CdS and (b) wz-CdS.
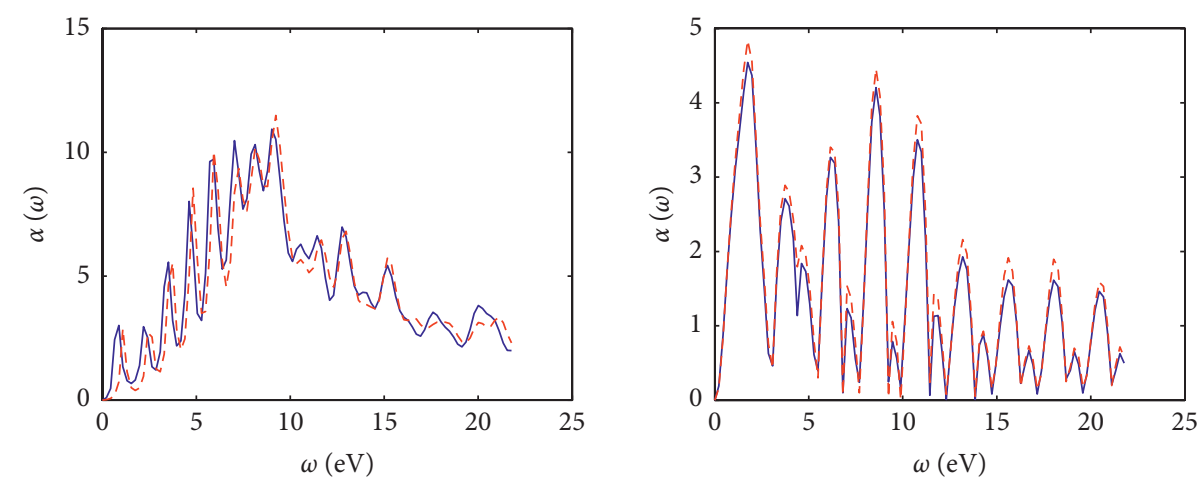

- LDA

- LDA

-. $\mathrm{PBE}$

(a)

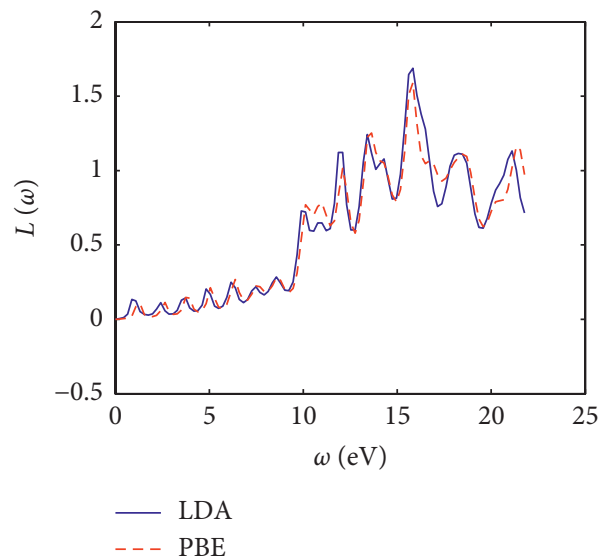

(c)

(d)

Figure 5: Absorption coefficients for (a) zb-CdS and (b) wz-CdS and energy loss spectrum for (c) zb-CdS and (d) wz-CdS. 
imaginary part of the dielectric function has its minima as it is expected.

The absorption coefficient and the energy loss function spectrum for $\mathrm{zb}$ - and $\mathrm{wz}-\mathrm{CdS}$ structure are calculated from frequency-dependent dielectric function using equation (3). The energy loss spectrum and absorption coefficient are shown in Figure 5.

Figures 5(a) and 5(b) show plot for optical absorption of zb- and wz-CdS based on LDA and PBE approximations. Absorption traits shown in Figures 5(a) and 5(b) represent energy-dependent spectra of absorption where a viable increasing and decreasing trend can be observed. Many peaks correspond to different electronic transitions from the valence band to the conduction band. Moreover, the oscillatory trend in these spectrums represents many-body interactions and is dependent on the strength of transitions. It is caused by the random orientation of the transition dipoles with applied electromagnetic field direction. The maximum values of the absorption coefficient are 10.93 and 11.49 for zb-CdS with respect to LDA and PBE approximations, respectively. However, the maximum values of absorption coefficients for wz-CdS are 4.54 and 4.84 using LDA and PBE approximations in that order.

Figures 5(c) and 5(d) show the value of energy loss evaluated using equation (3). The energy loss spectrum is important for describing the energy loss of fast electrons traversing the $\mathrm{zb}$ or $\mathrm{wz}$ phase of CdS. The peaks that occurred in both phases of $\mathrm{CdS}$ compound are believed to originate from the resonance of the $S 2 p$ valence electrons, possibly owing to the plasmon-like collective excitation of the valence and semicore electrons. All the peaks of $L(\omega)$ (Figures 5(c) and 5(d)) correspond to the rapid reduction in reflectance. The energy loss function $L(\omega)$ measures the loss of energy while propagating through the material. However, the absorption coefficient describes the transition of electrons from the valence band to the conduction band.

\section{Conclusions}

The structural, electronic, and optical properties of $\mathrm{zb}$ - and wz-CdS are studied using density functional theory. The exchange-correlation functional is approximated using LDA, GGA/PBE, GGA + U, and the hybrid functional (PBE0). The optimized lattice constants of $\mathrm{zb}$ - and wz-CdS with respect to these approximations are in good agreement with the experimental and previous theoretical values. To understand the electronic properties of $\mathrm{zb}$ - and wz-CdS structures, we have performed band structure calculation along the high symmetry of the Brillouin zone. The obtained bandgap values of $\mathrm{zb}$ - and wz-CdS in accordance with LDA and PBE potential are underestimated due to their poor approximation of exchange-correlation functional. However, the Hubbard correction to GGA and the hybrid functional approximation give a bandgap value which is consistent with the experimental results. The optical properties such as the real and imaginary parts of the dielectric function, the absorption coefficient, and the energy loss function are studied as a function of photon energy. The real part of the dielectric function is related to the transmission of photons within the material. However, the imaginary part of the dielectric function describes the absorption coefficient. The energy loss function shows the lost energy while the electromagnetic wave traverses inside both phases of CdS. The absorption coefficient describes the transition of electrons from the valence band to the conduction band from different orbitals.

\section{Data Availability}

The data used to support the findings of this study are included within the article.

\section{Conflicts of Interest}

The authors declare that there are no conflicts of interest regarding the publication of this article.

\section{Acknowledgments}

One of the authors Teshome Gerbaba expresses his thanks and appreciation to the Department of Physics, Wollega University, for its material support during this study.

\section{References}

[1] D. Keita, T. Yoshihiko, and M. Yoshikazu, "Physics and Chemistry review of layered Chalcogenide superconductors," Science and Technology of Advanced Materials, vol. 13, no. 5, pp. 1-11, 2012.

[2] S. Z. L. Abdulwahab, Y. Al-Douri, U. Hashim, and N. M. Ahmed, "Structural, analysis and optical studies of cadmium sulfide nanostructured," Procedia Engineering, vol. 53, pp. 217-224, 2013.

[3] L. I. Berger, Semiconductor Materials, CRC Press, New York, NY, USA, 1st edition, 1997.

[4] G. Melek and G. Emre, "Elastic, mechanical and phonon behavior of wurtzite cadmium sulfide under pressure," Crystals, vol. 7, no. 6, p. 164, 2017.

[5] Z. Oleg, R. Angel, and M. L. Cohen, "Calculated structural and electronic properties of CdSe under pressure," Physical Review B, vol. 51, no. 8, pp. 4926-4930, 1994.

[6] D. Soumendu, S.-D. Tanusri, and D. D. Sarma, "Wannier function study of the relative stability of zinc-blende and wurtzite structures in the $\mathrm{Cd} X(X=S$, Se, Te) series," Journal of Physics: Condensed Matter, vol. 20, Article ID 445217, no. 44, pp. 1-8, 2008.

[7] J. Frenzel and J.-O. Joswig, "Electronic-structure calculation of large cadmium chalcogenide nanoparticles," physica status solidi (b), vol. 249, no. 2, pp. 384-391, 2012.

[8] J.-O. Joswig, M. Springborg, and G. Seifert, "Structural and electronic properties of cadmium sulfide clusters," The Journal of Physical Chemistry B, vol. 104, no. 12, pp. 2617-2622, 2000.

[9] S. G. Thoma, A. Sanchez, P. P. Provencio, B. L. Abrams, and J. P. Wilcoxon, "Synthesis, optical properties, and growth mechanism of blue-emitting CdSe nanorods," Journal of the American Chemical Society, vol. 127, no. 20, pp. 7611-7614, 2005.

[10] P. Gopal, M. Fornari, S. Curtarolo et al., "Improved predictions of the physical properties of $\mathrm{Zn}$ - and $\mathrm{Cd}$-based wide 
band-gap semiconductors: A validation of the ACBN0 functional," Physical Review B, vol. 91, no. 24, pp. 1-9, 2015.

[11] L. Huang and J. G. Lu, "Synthesis, characterizations and applications of cadmium chalcogenide nanowires: A review," Journal of Materials Science \& Technology, vol. 31, no. 6, pp. 1-17, 2015.

[12] A. Oliva, "Formation of the band gap energy on CdS thin films growth by two different techniques," Thin Solid Films, vol. 391, no. 1, pp. 28-35, 2001.

[13] R. Lozad-Morales, O. Zelaya-Angel, and G. Torres-Delgado, "Photoluminescence in cubic and hexagonal CdS films," Applied Surface Science, vol. 175-176, pp. 562-566, 2001.

[14] U. Rossow, T. Werninghaus, D. R. T. Zahn, W. Richter, and K. Horn, "Thin epitaxial films of wide gap II-VI compounds studied by spectroscopic ellipsometry," Thin Solid Films, vol. 233, no. 1-2, pp. 176-179, 1993.

[15] Y.-W. Cheng, F. L. Tang, H.-T. Xue, H.-X. Liu, B. Gao, and Y.-D. Feng, "First-principles study on electronic properties and lattice structures of $\mathrm{WZ}-\mathrm{ZnO} / \mathrm{CdS}$ interface," Materials Science in Semiconductor Processing, vol. 45, pp. 9-16, 2016.

[16] H. X. Liu, F.-L. Tang, H.-T. Xue et al., "Lattice structures and electronic properties of WZ-CuInS2/WZ-CdS interface from first principles," Chinese Physics B, vol. 25, Article ID 123101, no. 12, pp. 1-10, 2016.

[17] H.-X. Deng, S. H. Li, J. Li, and S. H. Wel, "Effect of hydrogen passivation on the electronic structures," Physical Review B, vol. 85, pp. 1-5, 1958.

[18] G. Hautier, A. Jain, and S. P. Ong, "From the computer to the laboratory: materials discovery and design using first-principles calculations," Journal of Materials Science, vol. 47, no. 21, pp. 7317-7340, 2012.

[19] V. I. Anisimov, F. Aryasetiawan, and A. I. Lichtenstein, "Firstprinciples calculations of the electronic structure and spectra of strongly correlated systems: The LDA + U method," Journal of Physics: Condensed Matter, vol. 9, no. 4, pp. 767-808, 1997.

[20] T. B. Pryitno and R. Fahdiran, "Controlling band gap of monolayer $\mathrm{MnCl}_{2}$ with $\mathrm{LDA}+U$," Journal of Physics: Conference Series, vol. 1402, Article ID a044081, pp. 1-4, 2019.

[21] J. Heyd, G. E. Scuseria, and M. Ernzerhof, "Hybrid functionals based on a screened Coulomb potential," The Journal of Chemical Physics, vol. 118, no. 18, pp. 8207-8215, 2003.

[22] E. C. Ekuma, L. Franklin, G. L. Zhao, J. T. Wang, and D. Bagayoko, "Ab-initio local density approximation description of the electronic properties of zinc blende cadmium sulfide (zb-CdS)," Physica B: Condensed Matter, vol. 406, no. 8, pp. 1477-1480, 2011.

[23] P. Giannozzi, "Advanced capabilities for materials modeling with QUANTUM ESPRESSO,” Journal of Physics: Condensed Matter, vol. 29, no. 46, pp. 1-31, 2017.

[24] P. Giannozzi, S. Baroni, N. Bonini et al., "QUANTUM ESPRESSO: A modular and open-source software project for quantum simulations of materials," Journal of Physics: Condensed Matter, vol. 21, Article ID 395502, no. 39, pp. 1-20, 2009.

[25] J. P. Perdew and A. Zunger, "Self-interaction correction to density-functional approximations for many-electron systems," Physical Review B, vol. 23, no. 10, pp. 5048-5079, 1981.

[26] J. P. Perdew, K. Burke, and M. Ernzerhof, "Generalized gradient approximation made simple," Physical Review Letters, vol. 77, no. 18, pp. 3865-3868, 1996.

[27] S. L. Dudarev, G. A. Botton, S. Y. Savrasov, C. J. Humphreys, and A. P. Sutton, "Electron-energy-loss spectra and the structural stability of nickel oxide: An LSDA + U study," Physical Review B, vol. 57, no. 3, pp. 1505-1509, 1998.
[28] D. Fritch, B. J. Moorgan, and A. Walsh, "Self-consistent hybrid functional calculation: Implications for structural, electronic, and optical properties of oxide semiconductors," Nanoscale Research Letters, vol. 12, no. 1, pp. 1-7, 2017.

[29] H. J. Monkhorst and J. D. Pack, "Special points for Brillouinzone integrations," Physical Review B, vol. 13, no. 12, pp. 5188-5192, 1976.

[30] V. K. Kamble and V. B. Pujari, "Electrical and Microstructural properties of cadmium chalcogenides: A comparative study," Materials Research Laboratory, vol. 5176 pages, 2015.

[31] E. Deligoz, K. Colakoglu, and Y. Ciftci, "Elastic, electronic, and lattice dynamical properties of CdS, CdSe, and CdTe," Physica B: Condensed Matter, vol. 373, no. 1, pp. 124-130, 2006.

[32] S. H. Wei and S.B. Zhang, "Structure stability and carrier localization in $\mathrm{CdX}(\mathrm{X}=\mathrm{S}, \mathrm{Se}, \mathrm{Te})$ semiconductors," Physical Review B, vol. 62, no. 11, pp. 6944-6951, 2002.

[33] O. Zakharov, A. Rubio, X. Blasé et al., "Quasiparticle band structures of six II-VI compounds: ZnS, ZnSe, ZnTe, CdS, CdSe, and CdTe," Physical Review B, vol. 50, no. 10780, 1994.

[34] Y.-M. Yu, K.-m. Kim, B. O, K.-S. Lee, Y. D. Choi, and P. Y. Yu, "Band gap energy and exciton peak of cubic CdS/GaAs epilayers," Journal of Applied Physics, vol. 92, no. 2, pp. 1162-1164, 2002.

[35] K-H Helwege and O Madelung, Numerical Data and Functional Relationships Science and Technology, LandoltBörnstein, New Series Group III, Vols. 17a and 22a, Springer, New York, NY, USA, 1982.

[36] J. M. Carcione, F. Cavallini, J. Ba, W. Cheng, and A. N. Qadrouh, "On the Kramers-Kronig relations," Rheologica Acta, vol. 58, no. 1-2, pp. 21-28, 2019.

[37] O. Madelung, Semiconductors: Data Hand book, Springer, New York, NY, USA, 2004.

[38] L. Li, W. Wang, H. Liu, X. Liu, Q. Song, and S. Ren, "First Principles Calculations of Electronic Band Structure and Optical Properties of Cr-Doped ZnO," The Journal of Physical Chemistry C, vol. 113, no. 19, pp. 8460-8464, 2009. 